\title{
Transient Three-Dimensional Startup Side Load Analysis of a Regeneratively Cooled Nozzle
}

\author{
Ten-See Wang* \\ NASA Marshall Space Flight Center, Huntsville, Alabama, 35812
}

The objective of this effort is to develop a computational methodology to capture the startup side load physics and to anchor the computed aerodynamic side loads with the available data from a regeneratively cooled, high-aspect-ratio nozzle, hot-fired at sea level. The computational methodology is based on an unstructured-grid, pressure-based, reacting flow computational fluid dynamics and heat transfer formulation, a transient $5 \mathrm{~s}$ inlet history based on an engine system simulation, and a wall temperature distribution to reflect the effect of regenerative cooling. To understand the effect of regenerative wall cooling, two transient computations were performed using the boundary conditions of adiabatic and cooled walls, respectively. The results show that three types of shock evolution are responsible for side loads: generation of combustion wave; transitions among free-shock separation, restricted-shock separation, and simultaneous free-shock and restricted shock separations; along with the pulsation of shocks across the lip, although the combustion wave is commonly eliminated with the sparklers during actual test. The test measured two side load events: a secondary and lower side load, followed by a primary and peak side load. Results from both wall boundary conditions captured the free-shock separation to restricted-shock separation transition with computed side loads matching the measured secondary side load. For the primary side load, the cooled wall transient produced restricted-shock pulsation across the nozzle lip with peak side load matching that of the test, while the adiabatic wall transient captured shock transitions and free-shock pulsation across the lip with computed peak side load $50 \%$ lower than that of the measurement. The computed dominant pulsation frequency of the cooled wall nozzle agrees with that of a separate test, while that of the adiabatic wall nozzle is more than $50 \%$ lower than that of the

"Technical Assistant, ER43, Thermal and Combustion Analysis Branch, Propulsion Structure, Thermal, and Fluids Analysis Division, Senior Member AIAA. 
measurement. The computed teepee-like formation and the tangential motion of the shocks during lip pulsation also qualitatively agree with those of test observations. Moreover, a third transient computation was performed with a proportionately shortened $1 \mathrm{~s}$ sequence, and lower side loads were obtained with the higher ramp rate.

\section{Nomenclature}

$C_{1}, C_{2}, C_{3}, C_{\mu}=$ turbulence modeling constants, $1.15,1.9,0.25$, and 0.09 .

$$
\begin{aligned}
& C_{p} \quad=\text { heat capacity } \\
& D \quad=\text { diffusivity } \\
& F_{y z} \quad=\text { integrated force in the lateral direction } \\
& H \quad=\text { total enthalpy } \\
& K=\text { thermal conductivity } \\
& k \quad=\text { turbulent kinetic energy } \\
& p \quad=\text { pressure } \\
& Q \quad \text { = heat flux } \\
& T=\text { temperature } \\
& t \quad=\text { time, } \mathrm{s} \\
& u_{i} \quad=\text { mean velocities in three directions } \\
& x=\text { Cartesian coordinates } \\
& \varepsilon \quad=\text { turbulent kinetic energy dissipation rate } \\
& \theta \quad=\text { energy dissipation contribution } \\
& \mu \quad=\text { viscosity } \\
& \mu_{t} \quad=\text { turbulent eddy viscosity }\left(=\rho C_{\mu} \mathrm{k}^{2} / \varepsilon\right) \\
& \Pi \quad=\text { turbulent kinetic energy production } \\
& \rho \quad=\text { density } \\
& \sigma \quad=\text { turbulence modeling constants } \\
& \tau \quad=\text { shear stress } \\
& \omega=\text { chemical species production rate }
\end{aligned}
$$




$$
\begin{array}{ll}
\text { Subscripts } & \\
r & =\text { radiation } \\
t & =\text { turbulent flow } \\
w & =\text { wall }
\end{array}
$$

\section{Introduction}

Nozzle side loads are potentially detrimental to the integrity and life of almost all launch vehicle engines. For example, side load problems have been found in $\mathrm{J} 2$ engine, ${ }^{1}$ Block-I Space Shuttle Main Engine (SSME), ${ }^{2}$ and recently, the Fastrac Engine. ${ }^{3}$ More recently, the European Vulcain engine ${ }^{4}$ and the Japanese LE-7A engine ${ }^{5}$ have also experienced side load difficulties. A better understanding of the mechanisms that contribute to side loads during engine transient operations must be attained and the predictive ability of which has to be developed. Unfortunately, current level in understanding the nozzle side load physics is still limited and the design methods are mostly empirical. The lack of a predictive capability may result in system level failures, and ultimately reduced life and increased weight for new engine systems. Subsequently, a detailed, general predictive methodology based on the computational fluid dynamics (CFD) appears to be the most promising.

Since the physics lead to nozzle side load are transient in nature, it was suggested that only transient CFD analysis can simulate the highly transient phenomenon. ${ }^{6}$ Two early transient numerical attempts ${ }^{7,8}$ have been reported for SSME and J2S nozzles, respectively. Unfortunately, although both captured the nozzle hysteresis phenomenon that is considered to be one of the basic characteristics of liquid rocket engine nozzles, the axisymmetric assumption precludes the capturing of any asymmetric flows. In addition, the hysteresis phenomenon was measured by axial forces which have nothing to do with the side forces. That leads to the notion that only transient and three-dimensional (3-D) CFD analyses can simulate any asymmetric flow physics. Fortunately, as the computer hardware and computational methodologies advance, the affordability and reliability of transient 3-D nozzle computations increase. Yonezawa, et al. ${ }^{9}$ made the first 3-D CFD startup side load prediction for the LE-7, LE-7A and CTP50-R5-L nozzles, assuming frozen flow, constant specific heat, linear ramp rate, and adiabatic wall boundary condition. Qualitatively, their result captured certain physics such as transition from free-shock separation (FSS) to restricted-shock separation (RSS), along with FSS and RSS oscillations inside the nozzle. However, 
quantitative results were less satisfactory. For example, only one peak side load of $30 \mathrm{kN}$ was predicted for LE-7A engine at a nozzle pressure ratio (NPR) of 40 , while two side load peaks were observed at NPRs of 40 and 90 , with the measured peak side load of more than $200 \mathrm{kN}$ occurring at NPR of 90 . In addition, the Mach disk was never captured when the nozzle was flowing full, yet it was observed during the hot-fire test.

As stated by Yonezawa, et $\mathrm{al}^{9}$, the coarseness of their grid density was a factor that contributed to the failure of capturing Mach disk when nozzle was flowing full. It was further speculated that some of their modeling assumptions, e.g., the frozen flow, constant specific heat, and linear ramp rate, may have altered their predicted aerodynamics in matching those of tests. A series of two-dimensional (2-D) and axisymmetric numerical studies on the effects of those assumptions were performed, ${ }^{10}$ and it was found that combustion and inflow ramp rate drastically affect the computed side load physics. Basically, combustion changes the species composition hence the specific heat distribution, or the temperature distribution, while inflow ramp rate affects the flow residence time and in turn the reaction rate. These two intertwining factors affect both Mach disk shape and the Mach disk wave propagation history, and ultimately affecting wall pressure distribution, thereby influencing side load magnitudes. Tomita, et al ${ }^{11}$ also demonstrated the importance of combustion on side load physics in a subscale combustion test. Furthermore, since LE-7A nozzle has a regeneratively cooled section, similar to those of SSME and J2S, followed by film-cooling in the nozzle extension, it was further speculated that the adiabatic wall assumption was invalid for the regeneratively cooled wall. Note that Nave and Coffey ${ }^{1}$ observed that colder walls tend to retard flow separation, and two steady-state, 2-D CFD analyses ${ }^{12,13}$ showed that thinner, cold wall boundary layer is less susceptible to separation than the thicker, hot boundary layer of an adiabatic wall. This means that adiabatic wall is likely not a suitable boundary condition for regeneratively cooled nozzle walls.

This effort develops a computational methodology to investigate the startup side load physics of a regeneratively cooled, high aspect ratio, full scale SSME nozzle, firing at sea level. The computational methodology is based on an unstructured-grid, pressure-based, reacting flow computational fluid dynamics and heat transfer formulation. Based on the lessons learned from the work of Yonezawa, et $\mathrm{al}^{9}$, finite-rate chemistry was turned on throughout to properly consider the heat release and its effect on thermal fluid properties; an engine system simulation was used to obtain a nominal $5 \mathrm{~s}$ sequence to best simulate the actual pressure ramp rate and species time-varying history; to understand the effect of regeneratively cooling on side load physic, two 3-D, transient computations were computed in parallel with adiabatic wall and cooled wall. Available test data and observations are employed to help interrogating the 
computed physics. Lastly, a third transient case was performed using a proportionately shortened $1 \mathrm{~s}$ sequence with the cooled wall to investigate the effect of ramp rate on side load physics.

\section{Computational Methodology}

\section{A. Computational Fluid Dynamics and heat transfer}

The computational methodology is based on a multi-dimensional, finite-volume, viscous, chemically reacting, unstructured grid, and pressure-based fluid dynamics and heat transfer formulation. Time-varying transport equations of continuity, species continuity, momentum, total enthalpy, turbulent kinetic energy, and turbulent kinetic energy dissipation were solved using a time-marching sub-iteration scheme and are written as:

$$
\begin{aligned}
& \frac{\partial \rho}{\partial t}+\frac{\partial}{\partial x_{j}}\left(\rho u_{j}\right)=0 \\
& \frac{\partial \rho \alpha_{i}}{\partial t}+\frac{\partial}{\partial x_{j}}\left(\rho u_{j} \alpha_{i}\right)=\frac{\partial}{\partial x_{j}}\left[\left(\rho D+\frac{\mu_{t}}{\sigma_{\alpha}}\right) \frac{\partial \alpha_{i}}{\partial x_{j}}\right]+\omega_{i} \\
& \frac{\partial \rho u_{i}}{\partial t}+\frac{\partial}{\partial x_{j}}\left(\rho u_{j} u_{i}\right)=-\frac{\partial p}{\partial x_{i}}+\frac{\partial \tau_{i j}}{\partial x_{j}} \\
& \frac{\partial \rho H}{\partial t}+\frac{\partial}{\partial x_{j}}\left(\rho u_{j} H\right)=\frac{\partial p}{\partial t}+Q_{r}+\frac{\partial}{\partial x_{j}}\left(\left(\frac{K}{C_{p}}+\frac{\mu_{t}}{\sigma_{H}}\right) \nabla H\right)+\frac{\partial}{\partial x_{j}}\left(\left(\left(\mu+\mu_{t}\right)-\left(\frac{K}{C_{p}}+\frac{\mu_{t}}{\sigma_{H}}\right)\right) \nabla\left(V^{2} / 2\right)\right)+\theta \\
& \frac{\partial \rho k}{\partial t}+\frac{\partial}{\partial x_{j}}\left(\rho u_{j} k\right)=\frac{\partial}{\partial x_{j}}\left[\left(\mu+\frac{\mu_{t}}{\sigma_{k}}\right) \frac{\partial k}{\partial x_{j}}\right]+\rho(\Pi-\varepsilon) \\
& \frac{\partial \rho \varepsilon}{\partial t}+\frac{\partial}{\partial x_{j}}\left(\rho u_{j} \varepsilon\right)=\frac{\partial}{\partial x_{j}}\left[\left(\mu+\frac{\mu_{t}}{\sigma_{\varepsilon}}\right) \frac{\partial \varepsilon}{\partial x_{j}}\right]+\rho \frac{\varepsilon}{k}\left(C_{1} \Pi-C_{2} \varepsilon+C_{3} \Pi^{2} / \varepsilon\right)
\end{aligned}
$$

A predictor and corrector solution algorithm was employed to provide coupling of the governing equations. A second-order central-difference scheme was employed to discretize the diffusion fluxes and source terms. For the convective terms, a second-order upwind total variation diminishing difference scheme was used. To enhance the temporal accuracy, a second-order backward difference scheme was employed to discretize the temporal terms. Details of the numerical algorithm can be found in Ref's 14-17.

An extended $k-\varepsilon$ turbulence model ${ }^{18}$ was used to describe the turbulence. A modified wall function approach was employed to provide wall boundary layer solutions that are less sensitive to the near-wall grid spacing. Consequently, the model has combined the advantages of both the integrated-to-the-wall approach and the conventional law-of-the-wall approach by incorporating a complete velocity profile and a universal temperature 
profile $^{19}$. A 7-species, 9-reaction detailed mechanism ${ }^{19}$ was used to describe the finite-rate, hydrogen/oxygen afterburning chemical kinetics. The seven species are $\mathrm{H}_{2}, \mathrm{O}_{2}, \mathrm{H}_{2} \mathrm{O}, \mathrm{O}, \mathrm{H}, \mathrm{OH}$, and $\mathrm{N}_{2}$.

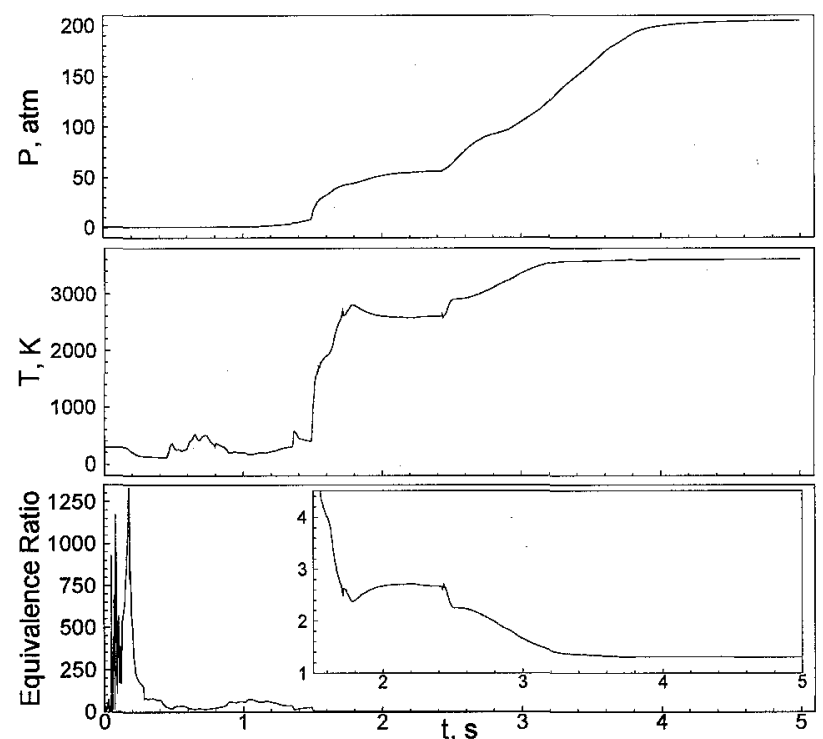

Fig. 1 Simulated thruster inlet properties during the startup transient.

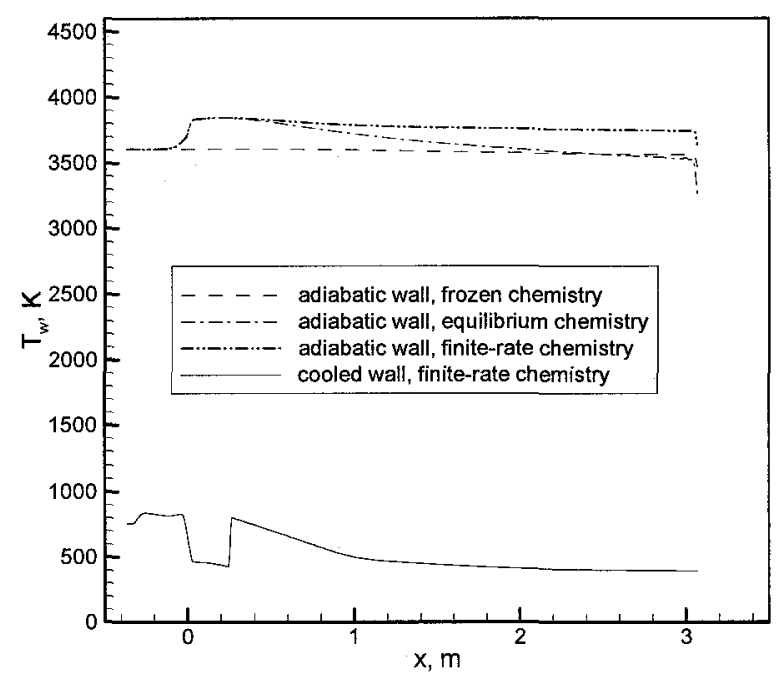

Fig. 2 A comparison of computed adiabatic wall temperatures and the regeneratively cooled wall temperature.

\section{B. Nominal 5 s Start-up Sequence}

The thruster inlet properties were obtained from an engine system calculation, simulating the effect of valve sequencing during a nominal $5 \mathrm{~s}$ operation. Figure 1 shows some of the inlet flow properties: the time-varying inlet pressure, temperature, and equivalence ratio profiles. Two significant pressure rise events can be identified. The first one occurs at $1.5 \mathrm{~s}$ due to oxygen prime, while the second one occurs at about $2.4 \mathrm{~s}$, caused by the step opening of the oxygen valves in the pre-burners. It can be seen that the thruster environment is fuel rich throughout the start-up transient, especially in the first $1.5 \mathrm{~s}$, setting up the potential for afterburning. The high equivalence ratio in the first $0.2 \mathrm{~s}$ is not significant since the flow rate is negligible.

\section{Wall Temperature Distribution for Regenerative Cooling}

For the SSME thrust chamber, the main combustion chamber (MCC) and the nozzle are cooled separately. MCC has 390 cooling channels, while the nozzle has 1080 cooling tubes. To model the two-way effect of the coolant flow from those channels and tubes, through the thruster chamber solid structures, onto the hot flow, is out of the scope of this 
effort. A simplified way is to apply a wall temperature distribution to the interior wall, calculated separately through a detailed thermal computation when nozzle is flowing full. Figure 2 shows the computed adiabatic and cooled wall temperatures. It can be seen that the effect of regenerative cooling cools the wall temperature to at least $2500 \mathrm{deg} . \mathrm{K}$ lower, which is not insignificant. Note that for the adiabatic wall transient, the wall temperatures are computed and not imposed. That is, only the wetted wall reaches the adiabatic temperature, while the un-wetted section stays cool. For the cooled wall transient, the computation started with adiabatic wall boundary condition, the cooled wall temperature distribution is only imposed at $1.5 \mathrm{~s}$ into the startup process, or when the pressure start to ramp significantly, as can be seen from Fig. 1.

\section{Computational Grid Generation}

Parametric studies conducted $^{14}$ show that a structured-cell dominated hybrid mesh performed more favorably than an unstructured-cell dominated hybrid mesh both in accuracy and efficiency, on flow physics and prediction of nozzle design parameters such as axial force and heat fluxes. Assuming that a grid suitable for axial force calculation is sufficient for side force calculation, the structured-cell dominated mesh $^{14}$ was used in this study and its layout is shown in Fig. 2. The structured (hexahedral) cells are used in the thruster and plume region, while the unstructured

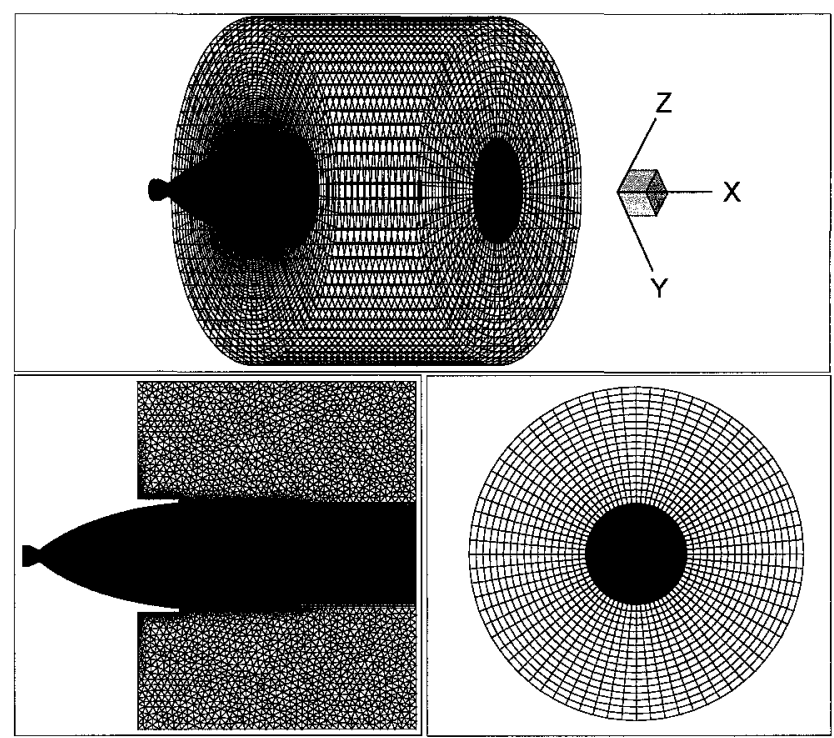

Fig. 3 The layout of hybrid grid 3d6. Top: an overall view. Bottom left: a cross-sectional cut through the nozzle axis. Bottom right: the exit plane. (prismatic) elements are used in the freestream region. The grid was generated using a software package GRIDGEN. ${ }^{20}$ The total number of points is $1,286,934$, which is considerably higher than the $145,500,145,500$, and 405,900 points used on LE-7, LE-7A, and CTP50-R5-L, ${ }^{9}$ respectively. Note that the SSME nozzle has a thrust optimized contour and its area ratio is 77.5. 


\section{Boundary and Inlet Conditions}

Fixed total condition was used for the outer boundary and a total pressure of $1 \mathrm{~atm}$ was used to simulate the nozzle hot-firing at sea level. No-slip condition was specified for the solid walls. The inlet flow properties obtained from the system simulation include the time varying total pressure, temperature, and propellant composition. The time varying propellant composition was preprocessed with the Chemical Equilibrium Calculation program ${ }^{21}$, assuming the propellants were ignited to reach equilibrium composition immediately beyond the injector faceplate. The fuel rich environment indicates the inlet composition contains mostly steam and excess hydrogen. At the start command, or time zero, the entire flowfiled is quiescent and filled with air. The axial wall temperature distribution ${ }^{14}$ of the cooled wall case was obtained from a separate conjugate heat transfer calculation.

\section{Results and Discussion}

The computations were performed on a cluster machine using 22 32 processors. Global time steps were varied throughout the computations: those of $2.5 \sim 10 \mu$ s were typically used during the initial transient and when the change

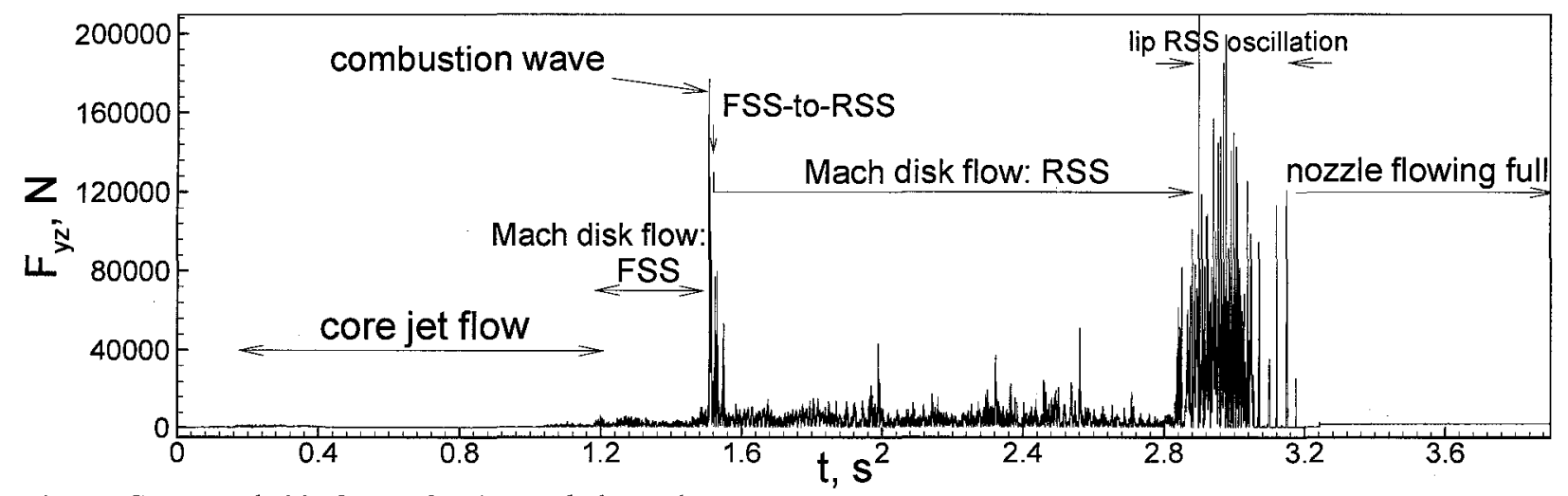

Fig. 4 Computed side forces for the cooled nozzle.

of flow physics was mild, and those of $1 \sim 2.5 \mu$ s were used when strong flow physics such as shock transitions and shock pulsations across the lip were occurring. The results of cooled wall case with the nominal $5 \mathrm{~s}$ sequence are presented first, then those of the adiabatic wall case with the same nominal $5 \mathrm{~s}$ sequence, followed by the results of another cooled wall case with a $1 \mathrm{~s}$ sequence. Finally the computed results are compared with available test data.

\section{A. The cooled wall case with the nominal 5 s sequence}


To compute the transient nozzle startup physics for a regeneratively cooled SSME hot-firing at sea level, the

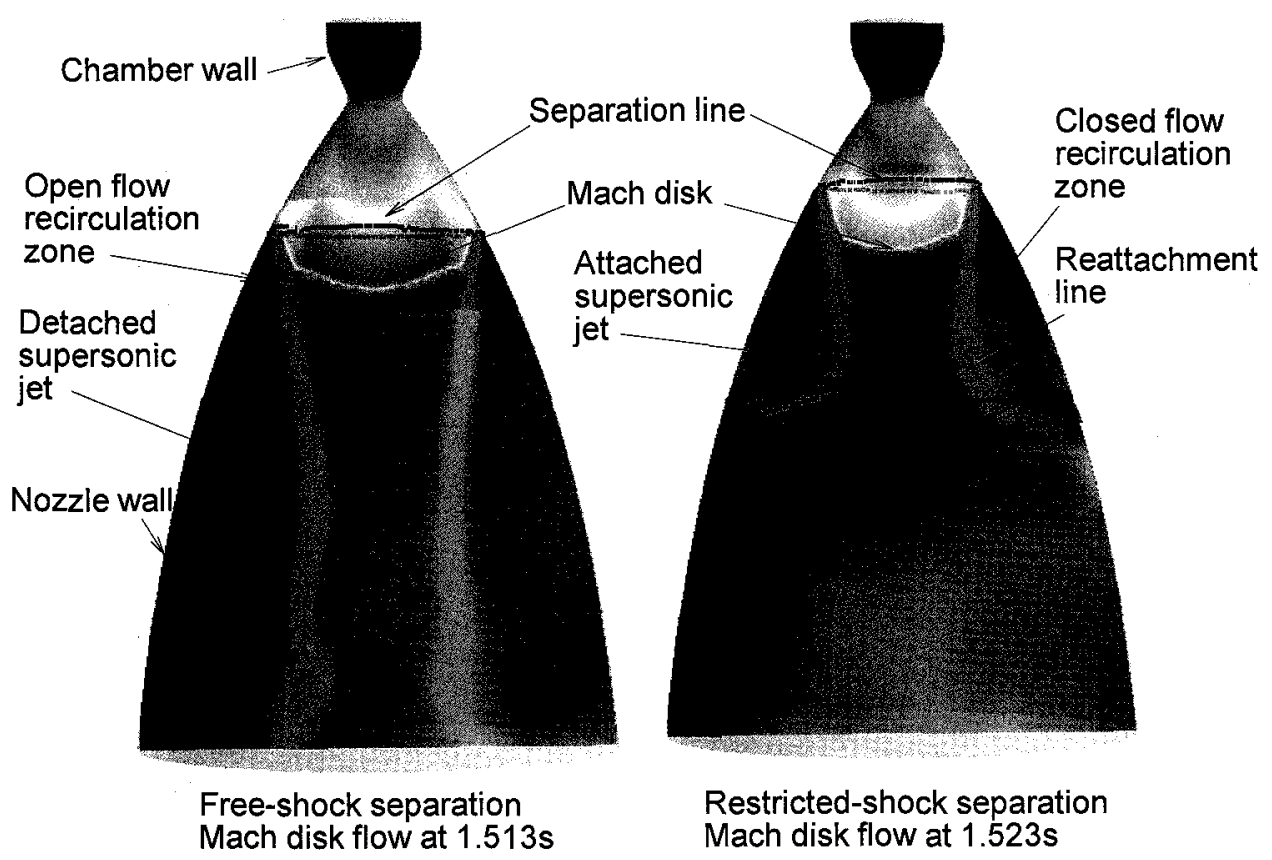

Fig. 5 Computed separation and reattachment line, and z-plane Mach number contours showing transition from free-shock separation to restricted-chock separation.

cooled wall temperature distribution shown in Fig. 2 is naturally the most realistic boundary condition, comparing to the adiabatic wall boundary condition. Figure 4 shows the computed time-varying side loads and associated physics, which are dictated by both the cooled wall boundary condition and the nominal $5 \mathrm{~s}$ startup sequence. It can be seen from Fig. 4, right after the start command, the initial side load is negligible and there is essentially no discernable flow physics. Until around $0.175 \mathrm{~s}$, a detached core jet ${ }^{10}$ comprising steam and hydrogen emerges from the throat. The core jet becomes stronger as the pressure increases. At $1.2 \mathrm{~s}$, the throat chokes and the core jet flow becomes a Mach disk flow. Unlike the situation captured in the 2-D planar SSME nozzle, ${ }^{10}$ while the core jet and subsequent Mach disk flow adhering to the wall due to the Coanda effect, ${ }^{22}$ creating asymmetric flow and producing early side forces; the core jet in the 3-D SSME nozzle is fairly centered, however, and produces negligible side forces during the core jet flow period. It is reasoned that the geometrical volume available for air pumping between the core jet and the wall is much larger in a high aspect ratio 3-D nozzle than that of a low aspect ratio 2-D nozzle, hence the difference. As a result, even as the Mach disk flow develops after $1.2 \mathrm{~s}$ and some small but detectable side load appears between 1.2 and $1.5 \mathrm{~s}$, the flow is still fairly centered. The Mach disk flow occurring in this period is in a 
FSS mode since the supersonic jet off the triple point is flowing freely and away from the wall. This is shown in the left figure of Fig. 5, where we can see the Mach disk, the detached supersonic jet off the triple point, the open flow recirculation zone, and a circular separation line which is the intersection of the upstream shock foot (stem) off the triple point and the nozzle wall. The solid wall created an adverse pressure gradient at the advancing Mach disk wave, causing the supersonic flow to separate.

At $1.5 \mathrm{~s}$ into the startup transient when the chamber temperature rises sharply (see Fig. 1), the composition of the Mach disk flow is essentially hydrogen-rich steam. That leads to afterburning in the mixing layer between the supersonic jet and the ambient air, raising temperature, as shown in the temperature contours at $1.503 \mathrm{~s}$ in Fig. 6. The combination of the fuel rich condition, the long residence time of the nominal $5 \mathrm{~s}$ sequence, create an environment for that elevated temperature front to quickly spread through the mixing layer, forming a fast expanding hot gas, as indicated in

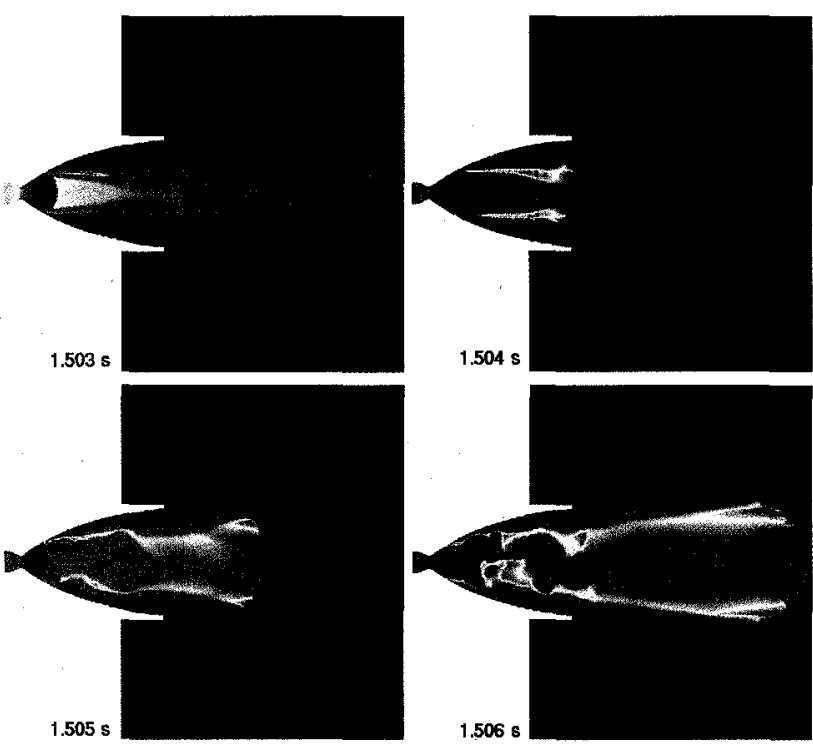

Fig. 6 Computed y-plane temperature contours of the cooled nozzle showing combustion wave. the rest of the plots in Fig. 6. Along side the fast expanding temperature wave, happening simultaneously, is a fast expanding pressure wave (not shown) called combustion wave. It also starts at the mixing layer inside the nozzle while propagating in all directions. A portion of the combustion wave moving inside the nozzle inevitably hits the wall, generating the first significant side load (Fig. 4) and several smaller ones thereafter due to shock reflections, while the rest expands away.

Soon after the disappearance of the combustion wave, the partially depleted hydrogen-rich jet gradually recovers and the Mach disk flow resumes its FSS mode. The afterburning continues, causing the supersonic jet to fluctuate and the Mach disk to move back and forth, while the entire Mach disk flow is adjusting itself to the changing nozzle contact area and the strengthening upstream flow. And then a physical change of the Mach disk flow soon follows. That is, the free-flowing supersonic jet of the FSS starts to move towards the wall at around $1.520 \mathrm{~s}$ and just attaches the wall at $1.523 \mathrm{~s}$ to form a close flow recirculation zone, as shown in the right figure of Fig. 5 . When the supersonic jet of the Mach disk flow is restricted to attaching the wall, the flow pattern is called a RSS. The 
phenomenon of a FSS transitioning to a RSS is called FSS-to-RSS transition. It can be seen from the right figure of Fig. 5 that when the supersonic jet is just fully attaching to the wall, the shape of the reattachment line is not circular, but resembling an asymmetric petal, exhibiting strong three-dimensionality. The transition from a rather symmetric wall pressure distribution of FSS (or the left figure in Fig. 5) to an asymmetric wall pressure distribution of RSS (or the right figure in Fig. 5), representing a large pressure disturbance and therefore causing a large side load jump in Fig. 4.

After the initial large side load jump due to the FSS-to-RSS transition, the remaining of the supersonic jet continues to becoming attaching to the wall and the recirculation bubble size shrinks, but the attachment line is still fairly asymmetric until around $1.530 \mathrm{~s}$, producing several smaller side load jumps, as shown in Fig. 4. Eventually the recirculation bubble size stabilizes, and the upstream shock stem, the supersonic jet, and the Mach disk form a Lambda shock. The supersonic jet, now only slightly longer than the upstream shock stem or foot, is called downstream shock stem or foot. The reattachment line

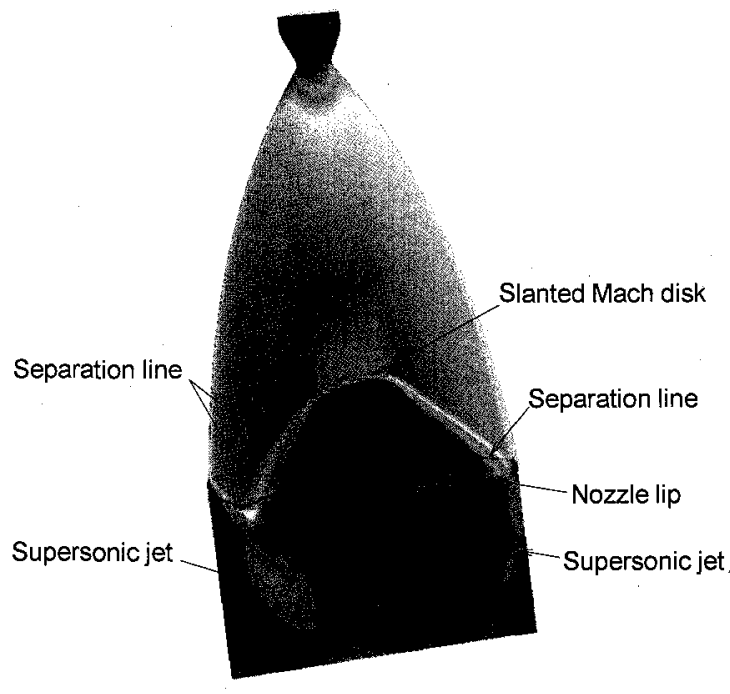

Fig. 8 Computed y-plane Mach number contours and separation line for the cooled nozzle during retracing portion of the shock pulsation across the lip at $3.099 \mathrm{~s}$.

is now more symmetric and the RSS walks the nozzle

wall downstream with its two shock feet, until the downstream foot reaches the nozzle lip at around $2.875 \mathrm{~s}$, as indicated in Fig. 4, while all the time the Mach disk and its two feet are oscillating back and forth due to the afterburning, producing occasional mild side load jumps that are lower in magnitude than that of the FSS-to-RSS transition.

Between $2.875 \mathrm{~s}$ and $3.15 \mathrm{~s}$ inside the transient, a unique side-load physics were captured for the first time. That is, the two-footed RSS Mach disk flow is computed to be pulsated or oscillated in-and-out of the lip several hundred times. The mostly symmetric two-footed RSS Mach disk flow goes asymmetric while crossing the nozzle lip, especially during the receding portion of the pulsation when the shock moves tangentially. This can be seen from Fig. 8 where the Mach number contours show a slanted Mach disk with asymmetric shock stems, resulting in a fatter 
supersonic jet on the left-hand side and a thinner supersonic jet on the right-hand side. In addition, two teepee-like flow recirculation zones marked by the separation line are showing above the nozzle lip, indicating the upstream shock foot (stem) has back-stepped into the nozzle at two places. This is a much more asymmetric and threedimensional shock pattern that that of the FSS-to-RSS transition shown in Fig. 5. In addition, unlike the FSS-to-RSS transition that happens only once at a location of small area ratio, this asymmetric side load physics happen hundred of times and near the lip where aspect ratio is at maximum, and for a very long time $(0.275 \mathrm{~s})$, resulting in the largest side load jump and the hundred of very large jumps for a long period of time, as shown in Fig. 4. After $3.15 \mathrm{~s}$, the nozzle is flowing full and the side load drops to negligible value.

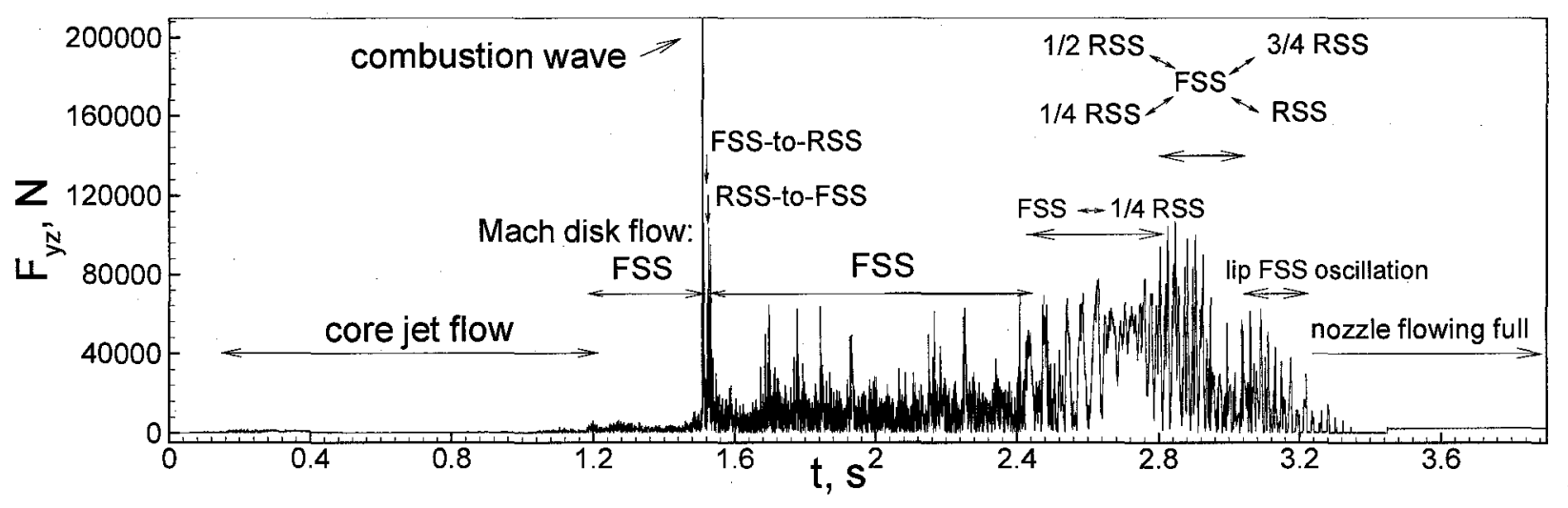

Fig. 9 Computed side forces for the adiabatic nozzle.

\section{B. The adiabatic wall case with the nominal $5 \mathrm{~s}$ sequence}

Next, we will examine the adiabatic wall case with the nominal $5 \mathrm{~s}$ sequence. The adiabatic wall boundary condition was used by all other computational studies, e.g., Ref. 8-9, to name a few. Figure 9 shows the computed time-varying side loads and associated physics for the adiabatic nozzle. It can be seen that like in the cooled wall case, the combustion wave and FSS-to-RSS transition also are computed, except the side load due to combustion wave is higher because of the implied energy loss in the cooled nozzle. The difference between the two wall boundary conditions starts after the FSS-to-RSS transition. For the adiabatic nozzle, after the FSS-to-RSS transition, the RSS lasted only $0.03 \mathrm{~s}$, after which RSS-to-FSS transition occurs (at $1.524 \mathrm{~s}$ ), producing a significant side load higher than that of its FSS-to-RSS transition, and the Mach disk flow stays as FSS until around $2.4 \mathrm{~s}$. This phenomenon may be explained again by the Coanda effect. That is, with a cooled wall, density is higher in the wall boundary layer. That leads to higher eddy viscosity, higher momentum, thinner boundary layer and lower pressure. 
Coanda effect then takes over, drawing the supersonic jet of the Mach disk flow to the wall. For an adiabatic wall where the temperature is stagnation temperature, the opposite is true. This computed flow physics agree with those reported in Ref's 12 and 13 in which thinner, cold-wall boundary was found to be less susceptible to separation than was the hot-wall, in steady nozzle flows. Between $1.524 \mathrm{~s}$ and $2.4 \mathrm{~s}$, the supersonic jet of the exhausting FSS Mach disk flow flaps, or fish tails, trying to become attached, to no avail. But with the second pressure rise event after 2.4 $\mathrm{s}$, the intensity of the fish tailing of the increases and the supersonic jet finally attaches to the wall, albeit partially in the circumferential sense, forming the simultaneous FSS and RSS, or partial RSS flow pattern. In particular, there are FSS $\leftrightarrow 1 / 4$ RSS transitions between 2.425 and $2.8 \mathrm{~s}$, as indicated in Fig. 9. The "1/4 RSS" means the attached region covering about a quarter of the circumference, while the rest of the region is detached. The FSS $\leftrightarrow 1 / 4$ RSS transition also represents many a back-and-forth transitions between FSS and 1/4 RSS. Figure 10 shows a snapshot of a typical $1 / 4$ RSS flow pattern in which the y-plane Mach number contours show a FSS flow pattern, but the z-plane contours

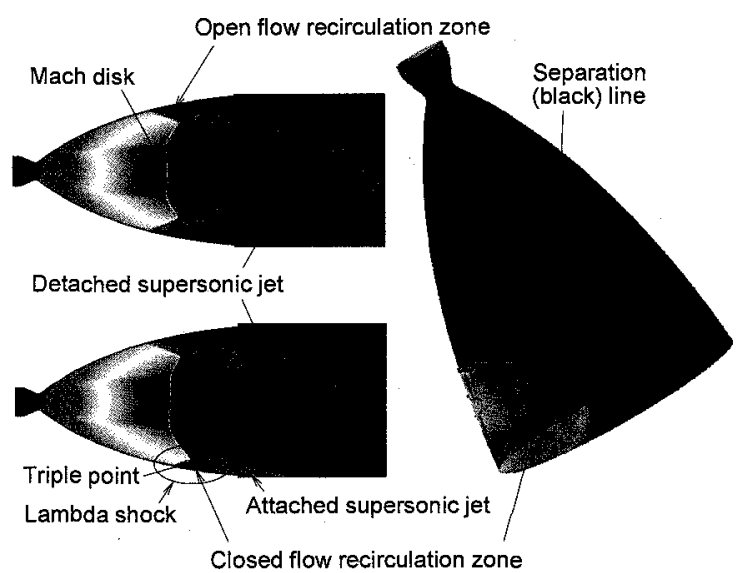

Fig. 10 Computed scalar contours of the adiabatic nozzle at 2.625s. Top left: Mach number contours on y-plane. Bottom left: Mach number contours on zplane. Right: wall $\mathrm{OH}$ concentration contours and separation line..

indicate an attached supersonic jet in the lower portion of the Mach disk flow, at $2.625 \mathrm{~s}$. The shock stem from the Mach disk side, the upstream shock foot, triple point, and the attached supersonic jet (downstream foot) constitute the Lambda shock formation, although only partially in the circumferential sense. The $1 / 4$ RSS flow pattern is asymmetric therefore generating higher side loads than those of FSS flow pattern occurring prior to $2.4 \mathrm{~s}$. Figure 10 also shows the wall $\mathrm{OH}$ concentration contours and separation line. Higher $\mathrm{OH}$ concentrations often indicate higher reaction rate or higher temperature. It can be seen that the visible $\mathrm{OH}$ concentration contours composed of two parts: the upper part shapes like a slanted plane that overlays with the upper part of the separation line; while the lower part coincides with the flow recirculation region but not quite coincides with the separation line that bounds the recirculation bubble. This is because the afterburning reaction is higher at both the separation line and the recirculated flow region where the temperatures are high. Plotting $\mathrm{OH}$ concentration contours is therefore an 
alternative way of indicating separation line. In addition, $\mathrm{OH}$ concentration contours clearly indicate the closed flow recirculation zone, slightly better than that of the separation line. Note the slanted plane in Fig. 10 closely resemble the so-called "tilted plane" as described in Ref. 1, which is the basis of several empirical side load equations. It needs to be pointed out though, when there is a slanted (separation) plane, there is the associated recirculation bubble. The "tilted plane" by itself does not represent the full physics happening in Fig. 10.

After $2.8 \mathrm{~s}$, the fish-tailing activity of the FSS intensifies and we have random transitions from FSS to various kinds of partial RSS, as shown in Fig. 9, and the side loads jump higher than those between $2.4 \mathrm{~s}$ and $2.8 \mathrm{~s}$. Then the fish-tailing activity drops off as the single shock foot of the FSS reaches the nozzle lip at around $3.04 \mathrm{~s}$. Between $3.04 \mathrm{~s}$ and $3.22 \mathrm{~s}$, the FSS Mach disk flow pulsates many times in-and-out of the nozzle lip, but the resulting side loads are lower than those of the RSS Mach disk flow pulsating across the lip. This is anticipated because the single shock-foot FSS causes less pressure disturbances than those of the double shock-foot RSS, when pulsating across the nozzle lip. The adiabatic nozzle is flowing full after $3.22 \mathrm{~s}$.

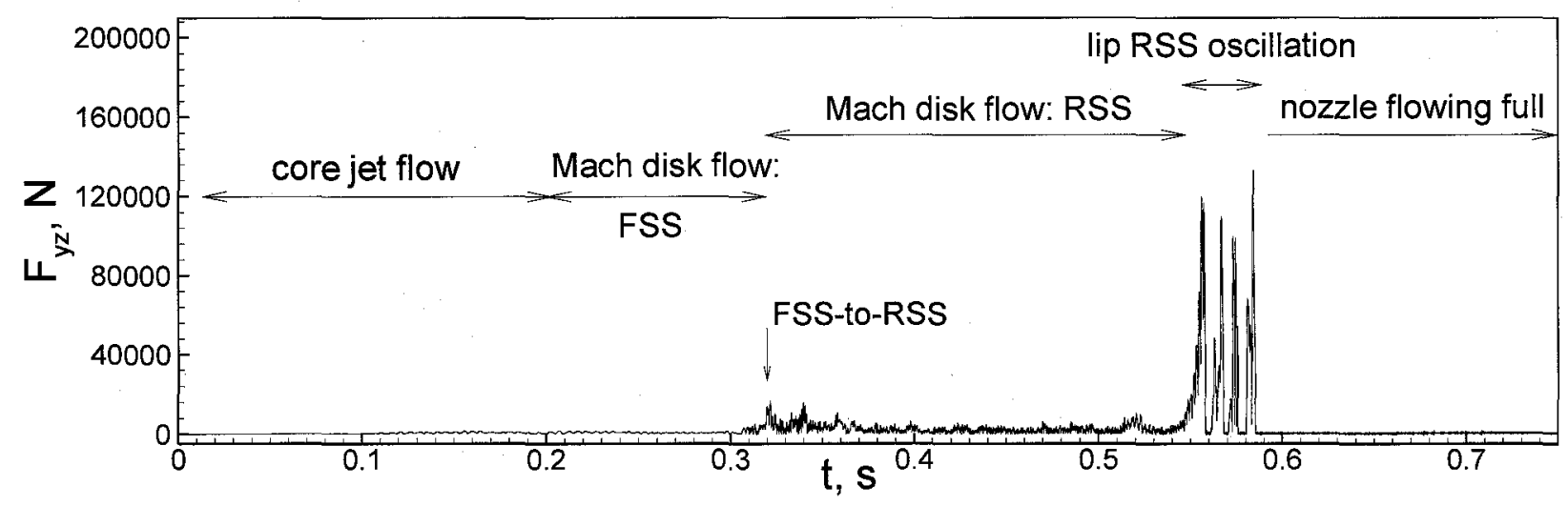

Fig. 11 Computed side load for the cooled wall case with a $1 \mathrm{~s}$ sequence.

\section{The cooled wall case with a $1 \mathrm{~s}$ sequence}

One of the important parameters that influence the side load physics is the ramp rate and ramp time. That is, the magnitude of the side load is not only a function of the chamber pressure, or the ratio of the chamber pressure to the ambient pressure, it is also a function of the rate of change of the chamber pressure and the total elapsed time when a specific side load is happening. The cooled wall case with a $1 \mathrm{~s}$ sequence is therefore performed. The $1 \mathrm{~s}$ sequence is achieved by taking the inflow properties of the nominal $5 \mathrm{~s}$ sequence shown in Fig. 1 and shrinking the total ramp 
time form the nominal $5 \mathrm{~s}$ to $1 \mathrm{~s}$. In terms of pressure history, e.g., this $1 \mathrm{~s}$ sequence goes through every absolute pressure plotted in Fig. 1, except it goes 5 times faster.

Figure 11 shows the computed side load history for the cooled wall with the aforementioned $1 \mathrm{~s}$ total ramp time. Comparing to the side load history for the cooled wall case with the nominal $5 \mathrm{~s}$ sequence, it can be seen that qualitatively they look similar, but there are several big differences. That is, the combustion wave is no where to be found, and the side loads caused by FSS-to-RSS transition and RSS pulsation across the lip are much smaller than those of the nominal $5 \mathrm{~s}$ sequence. When the ramp rate is

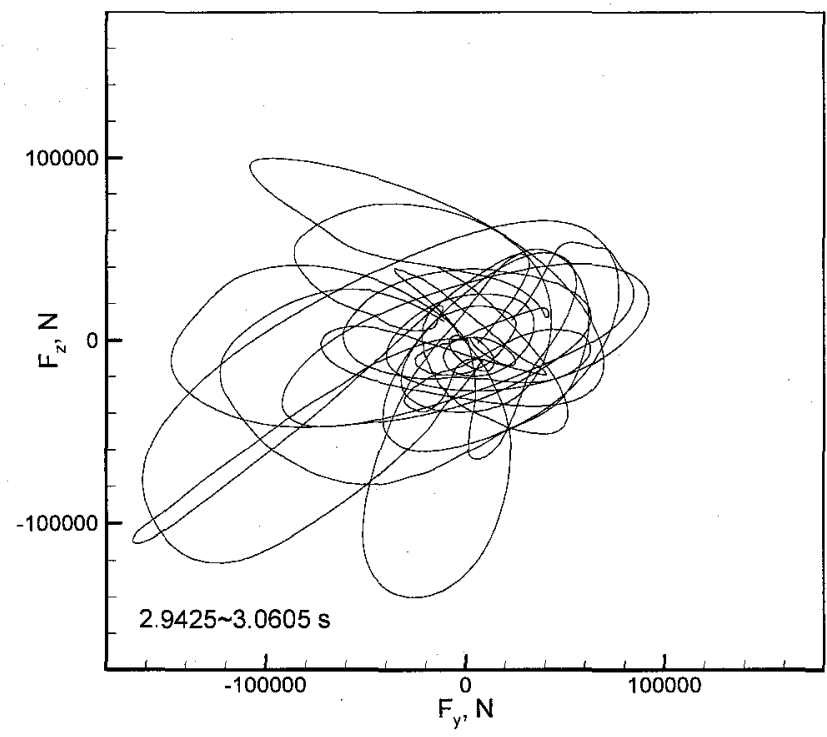

Fig. 12 Computed side force locus for the cooled nozzle. high and ramp time is short, there is not enough residence time for afterburning to be taken to a higher level and hence there is no combustion wave; the required time for FSS transitioning to RSS is shortened because the pressure increases faster, so that the induced side load is smaller; for RSS pulsation across the lip, both the reaction residence time and RSS lingering time are cut short, resulting in lowered side forces.

\section{Comparison of computational results with those of tests}

For both adiabatic and cooled nozzles with the nominal $5 \mathrm{~s}$ sequence, the shock pulsation across the lip shows tangential (or helical) motion of the side forces, during the retracing part, and not the exhausting part of the pulsation. These tangential motions are presented as polar plots, as shown in Fig's 12 and 13, respectively. The side force vector

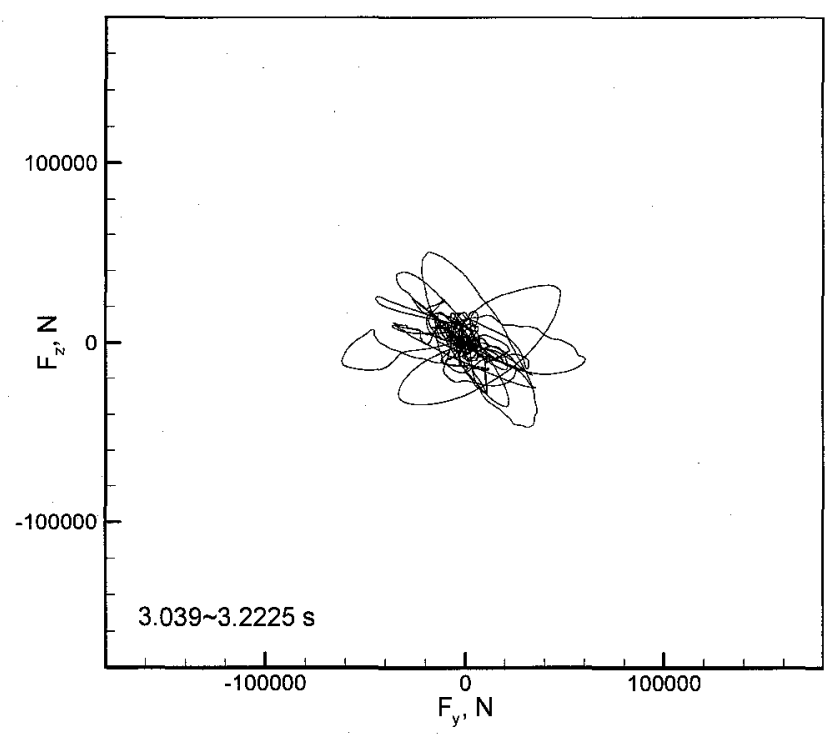

Fig. 13 Computed side force locus for the adiabatic nozzle. 
moves counter-clockwise (looking into the nozzle) during these tangential movements. It can be seen that the radii of the motion for the adiabatic nozzle is much restricted and centered around the origin, where the radii of the motion for the cooled nozzle cover significant more area and appears to be biased toward the lower-left quadrant, meaning the cooled nozzle has higher peak side load and significant tangential motion than those of the adiabatic nozzle, and the larger tangential motions do not centered around the geometrical center of the nozzle. It is noted that during this period, no teepee-like structure was captured with the adiabatic nozzle, while that was captured with the cooled nozzle, as shown in Fig. 8. The tangential shock motions were observed in both the SSME and J2S ${ }^{1}$ hotfirings.

As the shock pulses in and out of the nozzle exit plane, it is actually the shock legs attaching and detaching with the last part of the nozzle, resulting in pressure oscillations and generating side forces as shown in Fig. 4. Fig. 14 shows the computed wall pressure, shear stress, and heat flux histories for the cooled nozzle at a monitoring point near the nozzle lip. Those at the same axial location but circumferentially away from this point by 90,180 , and 270 degrees are qualitatively similar to these and are not shown. As mentioned above, with the Mach disk wave pulsating in-and-out of the nozzle, these flow properties fluctuate as the shock leg passing backand-forth by the monitoring point. Since gas temperature, density, and pressure are related, it is not surprising that the fluctuating histories of the wall pressure, shear stress and heat flux look qualitatively similar. In addition, the fact that the heat flux history appears to correlate with those of pressure and shear stress signifies the transient thermal load may also play a role in the total side

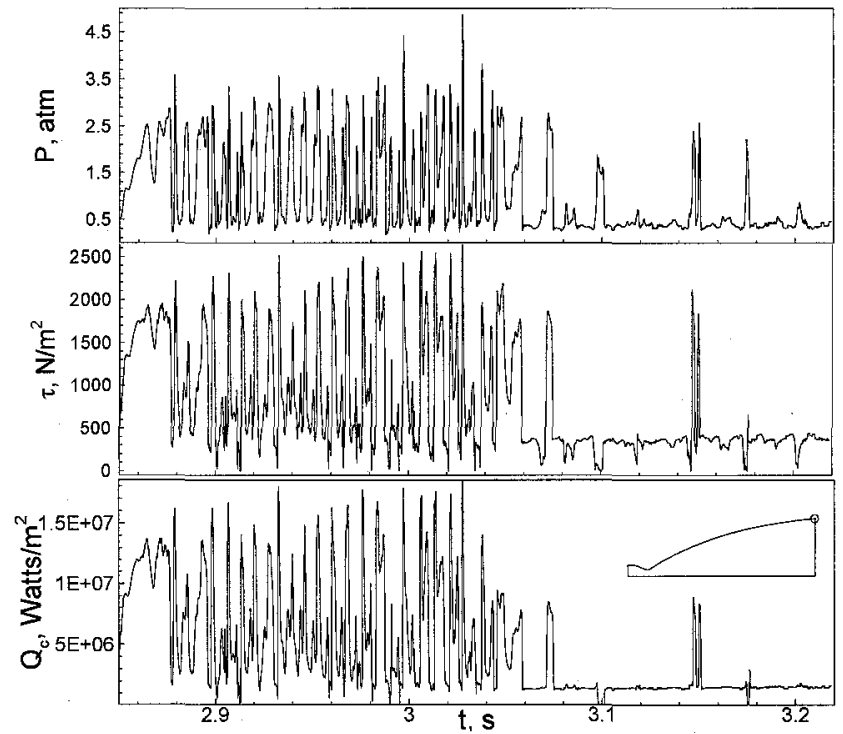

Fig. 14 Computed near lip wall pressure, shear stress, and heat flux histories for the cooled nozzle.

load physics. This is supported by the reported damage to some regenerative cooling tubes by strong heat-load during the start-up and shutdown processes in early LE-7A engine development, by Watanabe, et al. ${ }^{23}$. By looking at the fluctuating histories in Fig. 14, it can be seen that the frequencies of the fluctuations start slowly, slow down at about at $3 \mathrm{~s}$, and really slow down after $3.05 \mathrm{~s}$ into the start transient. Yet at a period from 2.9 to $3 \mathrm{~s}$, the fluctuating 
frequencies appear to be near a approximate steady-state. Hence, by clipping out the slower frequencies at both ends, a series of Fourier analyses were performed for pressure and heat flux histories between 2.90375 $\sim 3.0225 \mathrm{~s}$ for all four monitoring points such that dominant frequencies can be obtained to compare with those from a test. The result is presented as power spectral density profiles, as shown in Fig. 15. The dominant frequency based on the pressure is about $122 \mathrm{~Hz}$, while that based on the heat flux is about $125 \mathrm{~Hz}$, demonstrating the fluctuating frequencies between pressure and heat are indeed similar. As expected, the phases of the power density profiles are not exactly uniform for the four
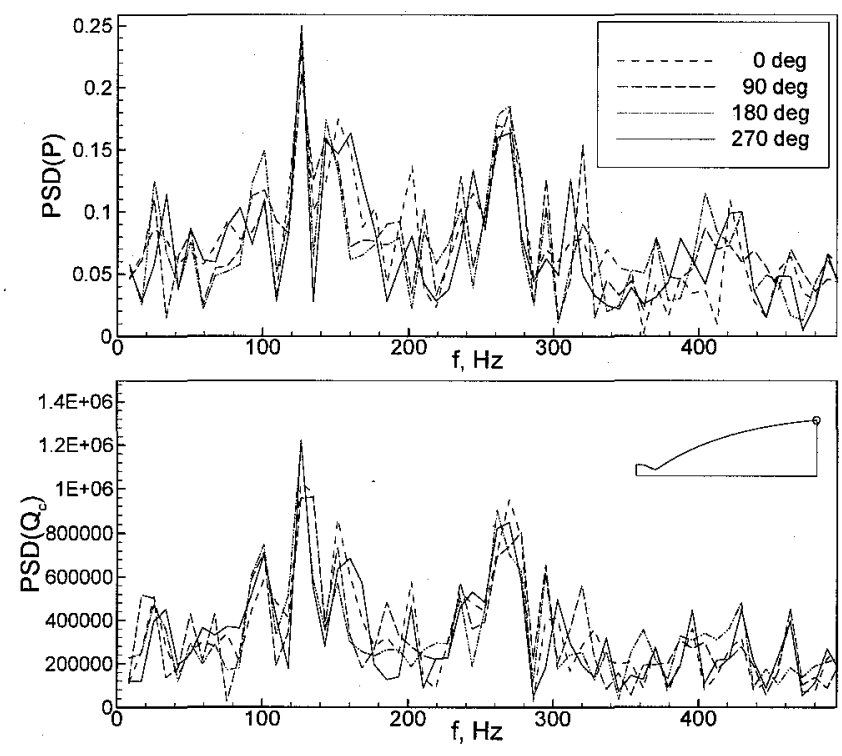

Fig. 15 Computed frequency domain for the cooled nozzle. monitoring points that are circumferentially 90 degrees apart. The same Fourier analyses were performed with computed wall pressure and temperature histories for the adiabatic nozzle between 3.04 and $3.2225 \mathrm{~s}$. The dominant frequency based on pressure is about $45 \mathrm{~Hz}$, while that based on temperature is slightly higher at about $49 \mathrm{~Hz}$.

Table 1 shows a comparison of the computed dominant frequencies for the cooled wall, and adiabatic nozzles, along with that of the test. The test data was scaled from a separate subscale air flow test to the SSME full scale conditions. It can be seen that the dominant frequencies computed from the cooled nozzle agree reasonably well with that of the test data, while those of the adiabatic nozzle are more than $50 \%$ too low.

Table 1. A comparison of dominant frequencies.

\begin{tabular}{|c|c|c|}
\hline & Dominant frequency, $\mathrm{Hz}$ & Variable \\
\hline \multirow{2}{*}{ Adiabatic nozzle } & 45 & pressure \\
\cline { 2 - 3 } & 49 & temperature \\
\hline Cooled nozzle & $\mathbf{1 2 2}$ & pressure \\
\cline { 2 - 3 } & $\mathbf{1 2 5}$ & heat flux \\
\hline Test data & 120 & pressure \\
\hline
\end{tabular}

Table 2 shows a comparison of the computed local peak side loads with the associated physics, and those from a hot-firing test. The test measured two local peak side loads. The first and lower local peak side load of $90 \mathrm{kN}$ occurs right after the first pressure-rise event or $1.5 \mathrm{~s}$ (see Fig. 1), while the second and the maximum side load of $200 \mathrm{kN}$ 
happens around $3 \mathrm{~s}$ into the nominal $5 \mathrm{~s}$ sequence. It can be seen that the computed local peak side load of $70 \mathrm{kN}$ due to FSS-to-RSS transition and that of $102 \mathrm{kN}$ due to RSS-to-FSS transition of the adiabatic nozzle, and that of 80 $\mathrm{kN}$ due to FSS-to-RSS of the cooled nozzle, are all agree reasonably well with the measured first peak side load. All those computed shock transitions occur around $1.5 \mathrm{~s}$ (Figs. 4 and 9), which agrees with the timing of the first peak measured from the test as well. The measured first local peak side load is therefore associated with the shock transitions and the computed local peak side loads due to shock transitions agree reasonably well with that of the test. It is noted that also occurring around $1.5 \mathrm{~s}$, the computed side loads due to combustion wave do not appear to be measured in the test. This is because precautionary measures such as the sparklers (not simulated in this study) are placed near the nozzle exit to burn off excess fuel flowing into the nozzle (see the equivalence ratio plot in Fig. 1), such that potentially detrimental situation is avoided. A flame torch is another method to burn off excess fuel that often used during hot-firing test of rocket engines.

Table 2. A comparison of local peak side loads.

\begin{tabular}{|c|c|c|c|l|}
\hline \multirow{2}{*}{$\mathrm{F}_{\mathrm{yz}}, \mathbf{k N}$} & \multirow{2}{*}{ Test } & \multicolumn{3}{|c|}{ Computation } \\
\cline { 3 - 5 } & & Adiabatic nozzle & Cooled nozzle & Responsible side load physics \\
\hline- & - & 395 & 176 & Combustion wave \\
\hline $\begin{array}{c}\text { secondary } \\
\text { peak }\end{array}$ & 90 & 70 & 80 & FSS-to-RSS transition \\
\hline $\begin{array}{c}\text { primary } \\
\text { peak }\end{array}$ & \multirow{2}{*}{200} & 102 & - & RSS-to-FSS transition \\
\cline { 3 - 5 } & & 110 & - & FSS-to-partial RSS transition \\
\cline { 3 - 5 } & & 60 & - & FSS pulsation across lip \\
\cline { 3 - 5 } & & - & 212 & RSS pulsation across lip \\
\hline
\end{tabular}

The measured second local peak side load of $200 \mathrm{kN}$, is more important than the first local peak side load, because it is the maximum side load that would cause the most damage to the hardware. The computed maximum side load of $212 \mathrm{kN}$ due to the RSS pulsation across the nozzle of the cooled nozzle agree with the measurement quite well and the computed timing of $2.9 \sim 3.0 \mathrm{~s}$ agree with that of the test also very well. However, although of the computed timing of $2.9 \sim 3.1 \mathrm{~s}$ of the second local peak side loads of the adiabatic nozzle also agree reasonably with that of the test, the magnitudes of $110 \mathrm{kN}$ due to FSS-to-partial RSS transition and $60 \mathrm{kN}$ due to FSS pulsation across the lip are too low and do not agree well with that of the measurement. The maximum side load is therefore associated with the RSS pulsation across the lip and the computed peak side load from the cooled nozzle agrees well with that of the test.

In summary, contrary to the intuition or results ${ }^{14}$ of axial force computation that cooled wall boundary condition 
gives lower axial thrust than that of adiabatic wall due to energy loss, the cooled nozzle produces much higher peak side force than that of the adiabatic nozzle. This is because the cooled wall promotes the Coanda effect, attracting the supersonic jet to stay attaching to the nozzle wall, thereby maintaining the RSS flow pattern after the FSS-toRSS transition, all the way through the RSS pulsation across the nozzle lip. The two-legged RSS pulsation across the lip in turn produces more wall pressure disturbance and flow asymmetry, than those of the one-legged FSS pulsation across the nozzle lip or the FSS-to-partial RSS transitions, thereby generating peak side load that agrees with the test measurement, while the adiabatic nozzle produces peak side load only about half as much. The twolegged RSS pulsation across the nozzle lip also produces dominant pulsation frequency agreeing with the test measurement, doubling that of the one-legged FSS pulsation across the nozzle lip.

\section{Conclusions}

A unique computational methodology based on a pressure-based computational fluid dynamics formulation and a system modeling for the transient inflow properties, was developed to predict the aerodynamic side load for regeneratively cooled, high aspect ratio nozzles. The computational methodology was anchored by simulating the full-scale SSME startup transient at sea level, with emphases on the wall thermal boundary condition and ramp time. Three types of shock evolution are computed to generate significant side loads that could cause hardware damages: the occurrence of combustion wave, shock transitions, and shock pulsations across the lip, although the side load induced by combustion wave can be avoided by installing excess fuel burning devices such as sparklers or flame torches. It is found that the wall thermal boundary conditions affect the computed side load physics. The cooled wall boundary condition promotes the Coanda effect that attracts the supersonic jet of the Mach disk flow to the wall to favor RSS flow pattern, while the adiabatic wall boundary condition does the opposite to repel the supersonic jet from the wall to support the FSS flow pattern, after the FSS-to-RSS transition. The computed first group of side loads for the FSS-to-RSS transition of the cooled wall nozzle, and FSS-to-RSS along with RSS-to-FSS transitions of the adiabatic wall nozzle, compare well with those of the secondary side load of the test. The computed peak side load due to RSS pulsation across the lip and the associated dominant pulsation frequency of the cooled wall nozzle agree well with the peak side load and the pulsation frequency of the test, respectively. However, the computed second group of side loads due to FSS-to-partial RSS transitions and FSS pulsation across the lip and the associated dominant pulsation frequency of the FSS pulsation are too low when compared to the primary side load and 
pulsation frequency of the test, respectively. The cooled wall boundary condition is therefore a more realistic boundary condition than that of the adiabatic wall, for a regeneratively cooled nozzle. The timings of the predicted side load peaks agree with those of the tests. In addition, when the ramp time is proportionately shortened from the nominal $5 \mathrm{~s}$ to $1 \mathrm{~s}$, not only the timings of the occurrence of the peak side loads are shortened, the magnitudes of the peak side loads are much lower, indicating the important role played by the ramp time and ramp rate on side load physics. Finally, since the side load induced by the FSS-to-RSS transition is considerably lower, the RSS oscillation across the lip along with its associated tangential shock motion appear to be the dominant side load physics for the regeneratively cooled, high aspect-ratio rocket engines.

\section{Acknowledgments}

This study was partially supported by a MSFC FY03 CDDF effort entitled "Nozzle Side Load Technology". The author wishes to thank Chief Aerodynamicist Werner Dahm for his guidance, and Dave Seymour for providing engine start-up simulation. Y.-S. Chen of Engineering Sciences, Inc. provided technical support and discussions. James Beck of Rocketdyne Boeing provided test observations on SSME side load physics. Discussions with Joe Ruf on side load physics, and with Daniel Dorney, and Kader Frendi of University of Alabama at Huntsville on frequency analysis are also acknowledged.

\section{References}

${ }^{1}$ Nave, L.H., and Coffey, G.A., "Sea Level Side Loads in High-Area-Ratio Rocket Engines," AIAA Paper 73-1284, Nov. 1973.

${ }^{2}$ Cikanek, H.A., "Characteristics of Space Shuttle Main Engine Failures," AIAA Paper 87-1939, June 1987.

${ }^{3}$ Brown, A.M., Ruf, J., Reed, D., D’Agostino, M.D., and Keanini, R., "Characterization of Side Load Phenomena Using Measurement of Fluid/Structure Interaction," AIAA Paper 2002-3999, July 2002.

${ }^{4}$ Hageman, G., Alting, J., and Preclik, D., "Scalability for Rocket Nozzle Flows Based on Subscale and Full-Scale Testing," Journal of Propulsion and Power, Vol. 19, No. 3, 2003, pp.321-331.

${ }^{5}$ Wantabe, Y., Sakazume, N., and Tsuboi, M., "LE-7A Engine Nozzle Problems During the Transient Operations," AIAA Paper 2002-3841, July 2002. 
${ }^{6}$ Hagemann, G., Terhardt, M., Frey, M., Reijasse, P., Onofri, M., Nasuti, F., and Ostlund, J., "Flow Separation and SideLoads in Rocket Nozzles," $4^{\text {th }}$ International Symposium on Liquid Space Propulsion, March 12-15, 2000, DLR Lampoldshausen.

${ }^{7}$ Wang, T.-S., "Numerical Study of the Transient Nozzle Flow Separation of Liquid Rocket Engines," Computational Fluid Dynamics Journal, Vol. 1, No. 3, 1992, pp. 319-328.

${ }^{8}$ Chen, C.L., Chakravathy, S.R., and Hung, C.M., "Numerical Investigation of Separated Flows," AIAA Journal, Vol. 32, No. 9, 1994, pp. 1836-1843.

${ }^{9}$ Yonezawa, K., Yokota, K., Tsujimoto, K., Sakazume, N., and Watanabe, Y., "Three-Dimensional Unsteady Flow Simulation of Compressed Truncated Perfect nozzles," AIAA Paper 2002-3991, July 2002.

${ }^{10}$ Wang, T.-S., "Transient Two-Dimensional Analysis of Side Load in Liquid Rocket Engine Nozzles," AIAA Paper 20043680, 11-14 July, 2004.

${ }^{11}$ Tomita, T., Sakamoto H., Onodera, T., Sasaki, M., Takahashi,M., Tamura, H., and Watanabe Y., "Experimental Evalualtion of Side-Load Characteristics on TP, CTP, and TO Nozzles," AIAA Paper 2004-3678, 11-14 July, 2004.

${ }^{12}$ Chang, C.L., Kronzon, Y., and Merkle, C.L., "Time-Iterative Solutions of Viscous Supersonic Nozzle Flows," AIAA Journal, Vol. 26, No. 10, 1988, pp. 1208-1215.

${ }^{13}$ Shimura K., Asako, Y., and Lee, J.H., "Numerical Analysis for Supersonic Flows in a Cooled Nozzle," Numerical Heat Transfer, Part A., Vol. 26, 1994, pp.631-641.

${ }^{14}$ Wang, T.-S., "Multidimensional Unstructured-Grid Liquid Rocket Engine Nozzle Performance and Heat Transfer Analysis," Journal of Propulsion and Power, Vol. 22, No. 1, January-February, 2006, pp. 78-84.

${ }^{15}$ Chen, Y.-S., Liu, J., Zhang, S., and Mallapragada, P., "An Integrated Tool for Launch Vehicle Base-Heating Analysis," Final Report, Engineering Sciences, Inc., Huntsville, AL, December 2001.

${ }^{16}$ Wang, T.-S., Chen, Y.-S., Liu, J., Myrabo, L.N., and Mead, F.B. Jr., "Advanced Performance Modeling of Experimental Laser Lightcraft," Journal of Propulsion and Power, Vol. 18, No. 6, 2002, pp. 1129-1138.

${ }^{17}$ Chen. Y.-S., Zhang S., and Liu, J., "Stage Separation Performance Analysis Project," Final Report, Engineering Sciences, Inc., Huntsville, AL, June, 2002.

${ }^{18}$ Chen, Y.-S., and Kim, S. W., “Computation of Turbulent Flows Using an Extended k- $\varepsilon$ Turbulence Closure Model," NASA CR-179204, Oct. 1987.

${ }^{19}$ Wang, T.-S., Droege, A., D'Agostino, M., Lee, Y.-C., and Williams, R., "Asymmetric Base-Bleed Effect on X-33 Aerospike Plume Induced Base-Heating Environment," Journal of Propulsion and Power, Vol. 20, No. 3, 2004, pp. $385-393$.

${ }^{20}$ Steinbrenner, J.P., Chawner, J.R., and Fouts, C., "Multiple Block Grid Generation in the interactive Environment," AIAA Paper 90-1602, June 1990. 
${ }^{21}$ Svehla, R.A., and McBride, B.J., "FORTRAN IV Computer Program for Calculation of Thermodynamic and Transport Properties of Complex Chemical Systems," NASA TN D-7056, Jan. 1973.

${ }^{22}$ Kumada, M., Mabuchi, I., and Oyakawa, K., "Studies on Heat Transfer to Turbulent Jets with Adjacent Boundaries," Bulletin of the Japan Society of Mechanical Engineers, Vol. 16, No. 100, 1973, pp. 1712-1722.

${ }^{23}$ Watanabe, Y., Sakazume, N., Yonezawa, K., and Tsujimoto, Y., "LE-7A Engine Nozzle Flow Separation Phenomenon and the Possibility of RSS Suppression by the Step inside the Nozzle," AIAA Paper 2004-4014, 11-14 July, 2004. 